\title{
Die personelle Verflechtung zwischen Sozialpartnern und Bundesregierung in Österreich, 1945-2015
}

\author{
Laurenz Ennser-Jedenastik1,* \\ 1 Institut für Staatswissenschaft, Universität Wien, Österreich \\ * E-Mail: laurenz.ennser@univie.ac.at
}

\section{Zusammenfassung}

Die personelle Verflechtung zwischen Parteien und sozialpartnerschaftlichen Organisationen ist einer der zentralen Gründe für die Stabilität des korporatistischen Institutionengefüges der Zweiten Republik. Dieser Beitrag liefert die bis dato umfangreichste und systematischste Analyse der personellen Verflechtungen zwischen der Bundesregierung und sozialpartnerschaftlichen Organisationen (Kammern und Gewerkschaften). Basierend auf biografischen Angaben zu allen 242 MinisterInnen und StaatssekretärInnen zwischen 1945 und 2015 zeigt sich, dass Sozialpartner-FunktionärInnen vor allem unter großen Koalitionen und Einparteienregierungen in Regierungsämter ernannt werden, und dass sie mit hoher Wahrscheinlichkeit in sozial- und wirtschaftspolitischen Ressorts tätig sind. Zwar schwankt der Verflechtungsgrad im Zeitverlauf, jedoch ist auch in jüngeren Jahrzehnten kein abnehmender Trend zu verzeichnen.

\section{Schlüsselwörter}

Sozialpartnerschaft, Korporatismus, Bundesregierung, Verflechtung, Österreich

\section{Personal linkages between social partnership organizations and the cabinet in Austria, 1945-2015}

\begin{abstract}
The personal linkages between political parties and social partnership organizations are a major contributor to the stability of corporatist institutions in postwar Austria. This paper presents the most comprehensive and systematic analysis to date of individual interlocks between the Austrian government and the corporatist institutions (chambers and trade unions). Drawing on biographical information for all 242 senior and junior ministers serving between I945 and 2015 , it shows that corporatist officials are more likely to be nominated to the cabinet under grand coalitions and single-party governments. They are also more likely to serve in portfolios dealing with matters related to social or economic policy. While the degree of interlock between cabinet and social partners varies over time, the data show no general decline in recent decades.
\end{abstract}

\section{Keywords}

social partnership, corporatism, cabinet, personal linkages, Austria

Dieser Beitrag entstand im Rahmen der Österreichischen Nationalen Wahlstudie (AUTNES), einem Nationalen Forschungsnetzwerk, finanziert vom Fonds zur Förderung der wissenschaftlichen Forschung (FWF) (SIO903-GII, Siogo3-Go8).

The author has declared that no competing interests exist. 


\section{Einleitung}

Die Sozialpartnerschaft ist untrennbar mit der politischen und wirtschaftlichen Geschichte der Zweiten Republik verbunden. Sie bildet gemeinsam mit der Vorherrschaft großer Koalitionen eine wesentliche Stütze der österreichischen Konsensdemokratie nach 1945. Zudem gilt sie zahlreichen wissenschaftlichen und anderen BeobachterInnen als Eckpfeiler des wirtschaftlichen Aufschwungs Österreichs in den I950er- und I960er-Jahren.

Zweifellos hat die Sozialpartnerschaft durch ihre Betonung von Verhandlungsprozessen und Kompromissfindung einen wesentlichen Beitrag zu sozialer Stabilität und wirtschaftlichem Wohlstand geleistet. Nicht zuletzt bot sie eine Arena der Kooperation für die beiden großen gesellschaftlichen Lager (das sozialistische und das katholischkonservative), die einander aufgrund der Erfahrungen der Ersten Republik und des Austrofaschismus noch feindlich gesinnt waren (Tálos 1985, 60).

Jedoch liegt die Bedeutung der Sozialpartnerschaft auch in ihrer engen Verflechtung mit dem politischen System, insbesondere mit den beiden (ehemaligen) Großparteien, SPÖ und ÖVP. Die Organisationen und Institutionen der Sozialpartnerschaft sind seit jeher von diesen beiden Parteien bzw. von spezifischen Teilorganisationen dieser Parteien dominiert und sowohl Sozialdemokratie als auch Volkspartei finden im Umfeld der Kammern und Gewerkschaften ein breites Rekrutierungsfeld für politisches Personal auf allen Ebenen.

Der vorliegende Beitrag untersucht eine spezifische Dimension der politischen Verflechtung, nämlich die Ernennung von Personen mit biografischem Hintergrund in der Sozialpartnerschaft (Kammern und Gewerkschaften) als MinisterInnen und StaatssekretärInnen. Dazu werden Informationen aus den Lebensläufen aller 242 Regierungsmitglieder im Zeitraum 1945 bis 2015 ausgewertet. Im Kern des Forschungsinteresses steht die Frage, welche Faktoren die Ernennung von Personen mit Sozialpartner-Hintergrund in Regierungsämter bestmöglich erklären können. Die Analyse formuliert Erwartungen auf Basis der Literatur bzw. der politischen Praxis und überprüft diese zum ersten Mal mit multivariaten Verfahren und für einen längeren Zeitraum als bisher untersucht wurde. Dadurch ist auch erstmals eine präzise relative Bewertung der Bedeutung von Faktoren auf Regierungs-, Parteien- und Ressortebene für die Ernennung von Personen mit SozialpartnerHintergrund möglich. Die vorliegende Arbeit leistet somit auch einen Beitrag zu Debatten über den (vermeintlichen) Niedergang der Sozialpartnerschaft und ihre Rolle vor dem Hintergrund der Europäischen Integration.

I Formal gesehen sind StaatssekretärInnen keine Mitglieder der Bundesregierung. Dennoch wird in diesem Beitrag der Begriff Bundesregierung verwendet, um die Gesamtheit aller MinisterInnen und StaatssekretärInnen zu bezeichnen.
Die Untersuchung beschränkt sich auf die Kern-Institutionen der Sozialpartnerschaft: den Österreichischen Gewerkschaftsbund (ÖGB), die Arbeiter- (AK), Wirtschafts- (WKO) und Landwirtschaftskammern (LWK). Nicht nur, dass es sich dabei um die größten und relevantesten korporatistischen Institutionen handelt, es sind in diesen Organisationen auch die Naheverhältnisse zu bestimmten politischen Parteien am deutlichsten ausgeprägt.

Die Analyse zeigt, dass (ehemalige) Sozialpartner-FunktionärInnen besonders unter großen Koalitionen und Einparteienregierungen ernannt und mit höherer Wahrscheinlichkeit in wirtschafts- und sozialpolitische Ressorts berufen werden. Interessanterweise spielt nach Kontrolle für den Regierungstyp das Vorschlagsrecht der Parteien für einzelne Ämter keine Rolle mehr für die Ernennung von Personen mit Sozialpartner-Hintergrund. Dieser Umstand liegt darin begründet, dass auch innerhalb der Regierungsteams von SPÖ und ÖVP in kleinen Koalitionen Sozialpartner-FunktionärInnen seltener zum Zug kamen (sowohl in absoluten als auch in relativen Zahlen).

\section{Sozialpartnerschaft in Österreich}

Die Sozialpartnerschaft ist eines der markantesten Charakteristika des österreichischen politischen Systems. Während tripartistische Verhandlungsmuster auch in einer Reihe anderer europäischen Demokratien zu finden waren und sind (Falkner/ Leiber 2004; Lehmbruch 1985) und ein spezifisches Merkmal konsensdemokratischer Systeme darstellen (Lijphart 1999, 37-38), ist Österreich im internationalen Vergleich jedenfalls als Extremfall einzustufen (Lehmbruch 1984; Siaroff 1999). In wenigen anderen Demokratien Europas ist die Bedeutung, Institutionalisierung und Stabilität sozialpartnerschaftlicher Arrangements ähnlich stark ausgeprägt (Kenworthy 2003).

Die österreichische Sozialpartnerschaft beschreibt ein institutionalisiertes Muster der Interessensvermittlung und Konzertierung zwischen den Dachverbänden der ArbeitgeberInnen und ArbeitnehmerInnen und der Bundesregierung. Sie war spätestens seit Ende der I950er-Jahre die zentrale Arena der Politikgestaltung in wirtschafts- und sozialpolitischen Fragen (Tálos 2006, 426; 2008, 26-27) und hat in diesen Themenbereichen hohe Relevanz (Krings 20I3; Tálos/Kittel 200I).

Ihre spezifische Ausbildung verdankt die Sozialpartnerschaft einer Reihe von begünstigenden Voraussetzungen (Tálos 2006, 429-43I). Dazu zählen etwa die starke Konzentration und Zentralisierung 
der relevanten Interessensorganisationen. ArbeitnehmerInnen und ArbeitgeberInnen sind in wenigen Dachverbänden organisiert, die über ein Vertretungsmonopol verfügen (im Fall der Kammern ist dieses durch die gesetzliche Pflichtmitgliedschaft abgesichert). Mit der politischen Dominanz von SPÖ und ÖVP in den Jahrzehnten nach 1945 ging zudem auch die politische Privilegierung der sozialpartnerschaftlich organisierten Interessensgruppen einher. Nicht zufällig fallen Herausbildung und Hochblüte der österreichischen Sozialpartnerschaft in die Zeit der schwarz-roten Nachkriegskoalitionen und der daran anschließenden Einparteienregierungen von ÖVP und SPÖ.

Im Zentrum dieser Arbeit steht ein weiterer begünstigender Faktor: die starke organisatorische und personelle Verflechtung zwischen den beiden (ehemaligen) Großparteien und den Kammern und Gewerkschaften. Die Tatsache, dass alle wichtigen sozialpartnerschaftlichen Interessensorganisationen von SPÖ oder ÖVP dominiert werden, hat zweifelsohne zur Stabilität der Sozialpartnerschaft beigetragen (Lehmbruch 1984).

Die wissenschaftliche Auseinandersetzung mit der Sozialpartnerschaft konstatiert seit mindestens drei Jahrzehnten einen krisenhaften Zustand bzw. eine Schwächung korporatistischer Muster in Österreich, befördert einerseits durch wirtschaftlichen Strukturwandel und den damit einhergehenden sinkenden gewerkschaftlichen Organisationsgrad (Blaschke 1999) sowie andererseits durch den Regierungswechsel von der großen Koalition (SPÖ-ÖVP) zu einer Mitte-Rechts-Regierung (ÖVP-FPÖ) im Jahr 2000 (Crepaz 1995; Gerlich I992; Gerlich et al. 1985; Karlhofer/Tálos 2000; Pernicka/ Hefler 2015; Prischnig 199I; Tálos 2006; Tálos/Fink 2003; Tálos/Stromberger 2004; Traxler 1987). Die Diagnose vom Niedergang der Sozialpartnerschaft ist aber nicht unwidersprochen geblieben (Compston 1994; Heinisch 2000; Kittel 1996; 2000), und besonders als Folge der Neuauflage der großen Koalition im Jahr 2007 wurde wieder ein gewisser Bedeutungsgewinn festgestellt (Obinger 2009).

Natürlich hat auch der Beitritt Österreichs zur Europäischen Union im Jahr 1995 Auswirkungen auf die Sozialpartnerschaft. Zum einen führt die Verlagerung von Kompetenzen auf die europäische Ebene zu geringeren Gestaltungsmöglichkeiten für nationale Akteure (so auch Interessensorganisationen und Verbände). Zum anderen eröffnet sich durch EU-weite Koordination von Interessensvertretungen ein neues politisches Aktionsfeld - in dem die Sozialpartner-Organisationen selbstverständlich nicht dieselbe privilegierte Stellung einnehmen wie im österreichischen Kontext (Karlhofer/ Tálos 1996; Schäfer/Streeck 2008). Während also die direkten Einflussmöglichkeiten der Sozialpartner durch den Prozess der Europäischen Integration geringer werden, bringt dieser Prozess gleichzeitig eine Aufwertung nationaler Regierungsmitglieder (als Entscheidungsträger im Rat der Europäischen Union) mit sich. Dadurch könnte die Bedeutung personeller Verflechtungen auf Regierungsebene für die korporatistischen Akteure wiederum steigen. Wiewohl der Einfluss der Europäischen Integration auf die Sozialpartnerschaft nicht im Zentrum dieser Arbeit steht, bildet er doch einen wesentlichen Kontextfaktor.

In der Literatur zur Sozialpartnerschaft wird - oft implizit - angenommen, dass der Verflechtungsgrad einen Indikator für den Einfluss der Sozialpartner auf die Politikgestaltung darstellt (Marko I992, 455). So es tatsächlich eine Korrelation zwischen der personellen Verflechtung zwischen Bundesregierung und Sozialpartnern und der Stärke korporatistischer Beziehungen insgesamt gibt, kann die vorliegende Arbeit auch einen Beitrag zur Diskussion über den vermeintlichen Niedergang der Sozialpartnerschaft leisten.

\section{Personelle und organisatorische Verflechtungen in der Sozialpartnerschaft}

Einer der Gründe für die langjährige Stabilität der österreichischen Sozialpartnerschaft ist die starke Verflechtung zwischen Interessensorganisationen (öffentlich-rechtlichen Kammern und Gewerkschaften) und politischen Institutionen (Parteien, Parlament und Bundesregierung) (Tálos 2006, 430). Der Österreichische Gewerkschaftsbund und die Arbeiterkammern weisen dabei starke personelle und organisatorische Verbindungen zur SPÖ auf ${ }^{2}$, während die Wirtschaftskammer Österreich und die Landwirtschaftskammern stark mit der ÖVP vernetzt sind. Als organisatorische Brückenköpfe zwischen Verbänden und Parteien dienen dabei die Gewerkschafts- bzw. Kammerfraktionen, die in den Parteien als Teilorganisationen agieren (Lehmbruch 1984; Müller 1994). Innerhalb der SPÖ handelt es sich dabei um die Fraktion sozialdemokratischer GewerkschafterInnen (FSG), innerhalb der ÖVP um den Österreichischen Wirtschaftsbund (ÖWB), den Österreichischen Bauernbund (ÖBB) und den Österreichischen Arbeitnehmerinnen- und Arbeitnehmerbund (ÖAAB) die drei wichtigsten Teilorganisationen der Volkspartei.

Die Verflechtung zwischen Parteien und Sozialpartnern wird in der Literatur ausführlich behandelt. Empirisch festgemacht wird sie für gewöhnlich an Phänomenen wie Kooptationen zwischen Gremien in Verbänden und Parteien, der Ämterkumulation bei SpitzenfunktionärInnen oder der Dominanz parteinaher Fraktionen bei Kammerwahlen. Lehmbruch (1984) etwa beschreibt

2 Eine Ausnahme bilden die Arbeiterkammern in Tirol und Vorarlberg sowie die Gewerkschaft Öffentlicher Dienst (GÖD), die allesamt von der Fraktion Christlicher Gewerkschafterinnen und Gewerkschafter (FCG) dominiert werden. 
die Delegationsrechte der FSG für Gremien innerhalb der SPÖ, die Kooptation von ÖGB- und AK-SpitzenfunktionärInnen in den SPÖ-Parteivorstand und die Tatsache, dass Gewerkschaftsvorsitzende traditionell Mandate im SPÖ-Parlamentsklub besetzen. ${ }^{3}$ Tálos (2008, 43) behandelt zudem noch die Dominanz der FSG bei Arbeiterkammerwahlen sowie jene des Wirtschaftsbundes bei Wirtschaftskammerwahlen und des Bauernbundes bei Landwirtschaftskammerwahlen (siehe dazu auch Karlhofer 2006). Fink (2006, 453) führt zudem noch die nicht unbeträchtlichen Geldflüsse zwischen Kammerfraktionen und Parteien an, wie sie auch bei Sickinger (2009, I60-I82) ausführlich dokumentiert sind.

Auf Individualebene ist Ämterkumulation das Resultat von organisatorischer Verflechtung, also die Kombination von öffentlichen politischen Ämtern, Parteifunktionen und Verbandsfunktionen. Das Ausmaß solch personeller Verflechtung wird oft anhand der Repräsentation von Sozialpartner-FunktionärInnen in der Bundesregierung und im Parlament festgemacht. Matis und Stiefel (1982) identifizieren bei rund jedem zweiten Mitglied des Nationalrates eine biografische Verbindung zu einer Interessensvertretung (wobei diese Zählung den Begriff „Interessensvertretung“ recht weit fasst). Diese Zahlen decken sich in etwa mit den Erkenntnissen von Marko (1992, 46I), der zudem zeigt, dass in den I980ern VertreterInnen der ArbeiternehmerInnen jenen der ArbeitgeberInnen im Nationalrat zahlenmäßig im Verhältnis zwei zu eins überlegen waren. Pelinka (I98I, I8) berichtet vergleichbare Zahlen für die I970er-Jahre.

Ebenso findet sich in der Literatur Zahlenmaterial über die Zugehörigkeit von Regierungsmitgliedern zu Verbänden der Sozialpartnerschaft. Gerlich (1985, II8) etwa stellt fest, dass der Anteil von VerbandsfunktionärInnen in der Bundesregierung zwischen 1956 und I983 von einem Drittel kontinuierlich auf etwa ein Fünftel zurückgegangen ist. Zum selben Schluss kommt Marko (1992, 465), der aber auch zeigt, dass nach der Wiederauflage der großen Koalition im Jahr 1987 der Funktionärsanteil in der Regierung bis Anfang der I990er auf nicht weniger als $50 \%$ anwächst. Anzumerken ist hier, dass Gerlich und Marko jeweils einen Datenpunkt in den Jahren 1970 und 1983 anführen, aber nicht klar ist, welche Bundesregierung dabei jeweils gemeint ist. In den Jahren der SPÖ-Mehrheitsregierungen unter Bruno Kreisky betrug der Anteil der VerbandsfunktionärInnen in der Bundesregierung hingegen meist deutlich über $40 \%$ (siehe unten).

Eine der empirisch fundiertesten Analysen zur Ämterkumulation in Regierungssystem und Sozialpartnerschaft findet sich bei Müller et al. (1994). Die AutorInnen zeigen etwa, dass die Verweildauer in Spitzenfunktio-

3 Eine Tradition, die nur unter dem SPÖ-Vorsitzenden Alfred Gusenbauer kurz eine Unterbrechung erfuhr. nen in den korporatistischen Institutionen bis zur Mitte der 1980er höher war als in den repräsentativen. Ab 1985 sinkt die durchschnittliche Verweildauer in beiden Institutionstypen. Des Weiteren nimmt das Gesamtausmaß der Ämterkumulation zwischen 1945 und 1990 langsam aber stetig ab.

\section{Hypothesen: Wie Sozialpartner-FunktionärInnen in Regierungsämter kommen}

Die Verflechtung zwischen Parteien, Regierungssystem und Verbänden wurde in der bisherigen wissenschaftlichen Auseinandersetzung fast ausschließlich deskriptiv behandelt. Diese Arbeit möchte darüber hinausgehen und fragt daher, welche Faktoren die bestehenden Verflechtungsmuster erklären können. Analytisch wird dabei auf der Individualebene angesetzt (d.h. die Beobachtungseinheiten sind einzelne MinisterInnen und StaatssekretärInnen), der empirische Fokus liegt auf der Bundesregierung.

Zur Hypothesenbildung wird nicht nur die Literatur zur österreichischen Sozialpartnerschaft herangezogen, sondern auch Forschung zur Besetzung von Ministerämtern (Bäck et al. 20II; Dowding/Dumont 20I5; Ecker et al. 2015). Dabei gliedern sich die Einflussfaktoren in drei Kategorien, nämlich Charakteristika der Regierungen, der Parteien und der zu besetzenden Regierungsämter.

\section{Charakteristika der Regierungen}

Zwei grundlegende Unterscheidungen in der vergleichenden Regierungslehre sind jene zwischen Mehrheits- und Minderheitsregierung sowie zwischen Einparteien- und Mehrparteienregierung. Während Minderheitsregierungen empirisch für Österreich wenig relevant sind (nur das Kabinett Kreisky I verfügte nicht über eine parlamentarische Mehrheit), so gab es doch eine längere Phase von Einparteienregierungen zwischen 1966 und 1983. Alle anderen Regierungen waren entweder große Koalitionen zwischen SPÖ und ÖVP oder kleine Koalitionen einer dieser Parteien mit der FPÖ. ${ }^{4}$

Welche Erwartungen knüpfen sich an diese drei Regierungstypen (Einparteienregierung, große Koalition, kleine Koalition)? Eine Möglichkeit zur Hypothesenbildung besteht darin, eine Rangordnung der Regierungstypen nach politischer Kongruenz mit den sozialpartnerschaftlichen Institutionen herzustellen. Anders gesagt geht es darum, ob die Grenzen zwischen Regierung und Opposition „innerhalb“ der Sozialpartnerschaft verlaufen. ${ }^{5}$ Da die Kammern und Gewerkschaften

4 Die Konzentrationsregierung Figl Ivon 1945 bis 1947 schloss auch die KPÖ mit ein, wird hier aber den großen Koalitionen zugeschlagen.

5 Natürlich befindet sich keine Partei per se „außerhalb“ der Sozialpartnerschaft. Die Formulierung bezeichnet hier Parteien, die - an- 
von SPÖ und ÖVP dominiert werden, sollte die politische Kongruenz bei großen Koalitionen am höchsten ausfallen. Hier sind beide wichtigen sozialpartnerschaftlich verankerten Player auch in der Regierung vertreten, die nicht sozialpartnerschaftlich verankerten Parteien befinden sich in Opposition. Die sozialpartnerschaftliche Logik verstärkt somit die parlamentarische Dynamik zwischen Regierung und Opposition. Nicht zuletzt war die Vorherrschaft großer Koalitionen nach 1945 ein für das Entstehen der Sozialpartnerschaft förderlicher Faktor (Müller 1985; Pelinka I98I).

Ein niedrigeres Niveau an Kongruenz herrscht bei Einparteienregierungen von ÖVP und SPÖ. Die Regierung wird von einer sozialpartnerschaftlich verankerten Partei gestellt, aber die Opposition setzt sich aus Parteien „innerhalb“ und „außerhalb“ der Sozialpartnerschaft zusammen. Bei kleinen Koalitionen ist die politische Kongruenz am geringsten. Der Konflikt zwischen Regierung und Opposition verläuft orthogonal zur Verankerung der Parteien in der Sozialpartnerschaft. Nicht zuletzt wurden die Schüssel-Kabinette (2000 bis 2007) oft als Tiefpunkt für die Sozialpartnerschaft angesehen (Rose 2000; Tálos 2008, 96). Das unterschiedliche Ausmaß politischer Kongruenz zwischen parlamentarischer und sozialpartnerschaftlicher Logik sollte sich selbst nach Kontrolle für die Partei, die ein Ministeramt besetzt - in unterschiedlichen Wahrscheinlichkeiten für die Berufung von Personen mit korporatistischem Hintergrund in die Regierung niederschlagen.

Hypothese 1: Die Ernennung von Regierungsmitgliedern mit sozialpartnerschaftlichem Hintergrund ist in großen Koalitionen am wahrscheinlichsten und in kleinen Koalitionen am unwahrscheinlichsten. Einparteienregierungen von ÖVP oder SPÖ fallen dazwischen.

\section{Charakteristika der Parteien}

Die Tatsache, dass SPÖ und ÖVP die Parteien mit den stärksten Bindungen an die sozialpartnerschaftlichen Institutionen sind, führt unmittelbar zu der Erwartung, dass diese Parteien bei Besetzungen von Ämtern in ihrem Verantwortungsbereich stärker auf Personal mit korporatistischem Hintergrund zurückgreifen sollten. Nicht, dass andere Parteien keine personellen Verbindungen mit Kammern oder Gewerkschaften hätten, ${ }^{6}$ aber das Ausmaß der Verflechtung ist bei SPÖ und ÖVP dennoch mit Abstand am höchsten. Das ist insofern wenig überraschend als auch die Literatur zu Verflechtung

ders als SPÖ und ÖVP - in Gewerkschaften und Kammern über nur schwache Verankerung verfügen.

6 Beispiele aufseiten der FPÖ sind etwa Mathias Reichhold als Vizepräsident der Kärntner Landwirtschaftskammer, Helmut Krünes als Kammerrat der Kammer für gewerbliche Wirtschaft Niederösterreich oder Sigisbert Dolinschek als Vorstandsmitglied der AK Kärnten. von Parteien und Interessensorganisationen traditionell starke Verbindungen zwischen Gewerkschaften und sozialdemokratischen Parteien einerseits sowie konservativen Parteien und Unternehmerverbänden andererseits identifiziert (Allern et al. 2007; Allern/Bale 20I2; Anthonsen et al. 20II; Aylott 2003; Otjes/Rasmussen 2015).

Hypothese 2 formuliert daher die Annahme, dass Ämter im Verantwortungsbereich von Sozialdemokraten oder Volkspartei mit höherer Wahrscheinlichkeit mit Sozialpartner-FunktionärInnen besetzt werden. ${ }^{7}$

Hypothese 2: Regierungsämter werden eher an Personen mit sozialpartnerschaftlichem Hintergrund vergeben, wenn das Amt in den Verantwortungsbereich von SPÖ oder ÖVP fällt.

Eine häufige Annahme in der Literatur zur Besetzung von Ministerämtern ist, dass Parteivorsitzende den größten Einfluss auf die Vergabe von Regierungsposten haben (Dowding/Dumont 2009; Indridason/Kam 2008). Bei aller realpolitischen Einschränkung dieser Annahme durch Begehrlichkeiten innerparteilicher Gruppierungen (Ennser-Jedenastik 20I3; Kam et al. 20IO) spielen Parteivorsitzende jedenfalls eine zentrale Rolle bei der Verteilung von Regierungsämtern.

Die Zuteilung von Ministerämtern stellt Parteivorsitzende vor ein klassisches Delegationsproblem (principal agent problem), das durch die Auswahl von Personal mit ähnlichen Präferenzen vermindert werden kann (Andeweg 2000; Indridason/Kam 2008). Daraus leitet sich die Hypothese ab, dass Parteivorsitzende mit sozialpartnerschaftlichem Hintergrund bevorzugt auf Personen zurückgreifen, die ebenfalls in korporatistischen Organisationen sozialisiert wurden.

Hypothese 3: Regierungsämter werden eher an Personen mit sozialpartnerschaftichem Hintergrund vergeben, wenn der oder die Parteivorsitzende der ressortverantwortlichen Partei einen sozialpartnerschaftlichen Hintergrund aufweist.

\section{Charakteristika des Amtes}

Nicht alle Regierungsämter sind gleich erstrebenswert. Manche Positionen bieten den InhaberInnen mehr Einfluss und Prestige, manche weniger. Darüber hinaus hat jedes Amt ein eigenes inhaltliches Profil. Aus der Forschung zur Bildung von Koalitionsregierungen ist bekannt, dass die inhaltlichen Präferenzen von Parteien

\footnotetext{
7 Diese Hypothese mag auf den ersten Blick trivial erscheinen, jedoch ist hier entscheidend, dass sie in Konkurrenz zum Faktor Regierungstyp (HI) getestet wird. Zudem soll nicht darauf vergessen werden, dass sich auch unter FPÖ/BZÖ-Regierungsmitgliedern Personen mit Verbindungen in Sozialpartner-Organisationen befanden.
} 
eine wesentliche Rolle bei der Verteilung von Ressorts spielen (Bäck et al. 20II; Browne/Feste 1975; Ecker et al. 2015). Sozialdemokratische Parteien besetzen meist sozialpolitische Ressorts, konservative Parteien hingegen bevorzugen etwa das Innen-, Außen- oder Verteidigungsministerium, während liberale Parteien oft nach dem Justiz- oder Finanzressort streben und grüne Parteien meist das Umweltministerium erhalten (Budge/ Keman 1990).

Für Personen aus dem sozialpartnerschaftlichen Bereich gilt, dass sie durch die Tätigkeit in Kammern oder Gewerkschaften jedenfalls in Berührung mit sozio-ökonomischen Themen gekommen sind und dort oft auch Expertenwissen gesammelt haben. Daher ist eine logische Erwartung, dass Personal mit korporatistischem Hintergrund bevorzugt in sozio-ökonomischen Fachressorts anzutreffen ist. Hinweise darauf gibt es auch in der einschlägigen Literatur. So erwähnen etwa Pelinka (198I, 19) und Schwediauer (20I0, 96) die Konvention, dass das Sozialministerium bei Regierungsbeteiligung der SPÖ für gewöhnlich mit einer Person aus dem Gewerkschaftsbund besetzt wird. ${ }^{8}$

Hypothese 4: Regierungsämter werden eher an Personen mit sozialpartnerschaftlichem Hintergrund vergeben, wenn das zu besetzende Ressort in den sozialoder wirtschaftspolitischen Bereich fällt.

Der sozial- und wirtschaftspolitische Bereich wird im Bezug auf $\mathrm{H}_{4}$ allerdings weit gefasst. Sozialpolitische Ressorts sind jene, deren Zuständigkeiten wesentliche Bereiche des Wohlfahrtsstaates abdecken (Soziales/Arbeit, Gesundheit und Familie). Wirtschaftspolitische Ressorts sind jene, die sich im Kern mit der Besteuerung, Verwaltung, Regulierung oder Förderung ökonomischer Aktivitäten auseinandersetzen (Finanzen, Wirtschaft/ Handel, Landwirtschaft sowie alle für Infrastruktur zuständigen Ministerien).

\section{Daten und Methode}

Die Datengrundlage für die Überprüfung der vier Hypothesen bildet die Gesamtheit aller MinisterInnen und StaatssekretärInnen der Zweiten Republik, beginnend mit dem Kabinett Figl I (d. h. ohne die Mitglieder der Provisorischen Staatsregierung unter Karl Renner). Es werden alle Personen berücksichtigt, egal ob sie zu Beginn oder während einer Regierungsperiode ernannt wurden. Insgesamt umfasst der Datensatz 242 Personen, wovon 20 Individuen für zwei zeitlich nicht unmittelbar aufei-

8 Die bisher einzige Ausnahme von dieser Regel bildet Erwin Buchinger, Sozialminister im Kabinett Gusenbauer, der vor seiner politischen Karriere im Arbeitsmarktservice tätig war. nanderfolgende Perioden im Amt waren. Tabelle I bietet einen Überblick über zentrale Charakteristika der Regierungsmitglieder.

Tabelle 1: Deskriptiver Überblick über die Daten

\begin{tabular}{ll}
\hline Charakteristikum & Anteil \\
\hline Frauen & $19 \%$ \\
MinisterInnen & $66 \%$ \\
SPÖ & $43 \%$ \\
ÖVP & $41 \%$ \\
FPÖ & $10 \%$ \\
Parteilos & $6 \%$ \\
Sozialpartner-Hintergrund & $29 \%$ \\
\hline
\end{tabular}

Anmerkung: N=242 Individuen. Ein KPÖ-Minister (Karl Altmann, 1945-1947) nicht berücksichtigt.

Wie aus Tabelle I hervorgeht, sind rund ein Fünftel der ernannten Personen Frauen, wobei der Frauenanteil im Verlauf der Zeit kontinuierlich gestiegen ist: Vor 1960 gab es kein einziges weibliches Regierungsmitglied, zwischen 1960 und 1979 betrug der Anteil I4 \%, zwischen I980 und I999 2I \% und zwischen 2000 und $201535 \%$. Von allen Personen mit Sozialpartner-Hintergrund sind nur I4 \% weiblich, während der Frauenanteil unter allen anderen Regierungsmitgliedern $20 \%$ beträgt.

Ziemlich genau zwei Drittel aller Individuen im $\mathrm{Da}-$ tensatz hatten Ministerämter inne, das verbleibende Drittel fungierte als StaatssekretärInnen. SPÖ und ÖVP stellten das Gros der ernannten Personen (jeweils über $40 \%$ ), ein Zehntel kam von der FPÖ und rund sechs Prozent waren parteilos.

Die mittlere Verweildauer im Amt beträgt 3,7 Jahre (Median), wobei hier extreme Varianz vorliegt. Einige Personen waren nur wenige Wochen im Amt (Michael Krüger etwa quittierte nach nur 25 Tagen den Dienst als Justizminister), während andere hohe Verweildauern aufweisen (an der Spitze Wolfgang Schüssel und Franz Löschnak mit Amtsperioden von über 17 Jahren). Personen mit Sozialpartner-Hintergrund weisen im Mittel eine etwas höhere Verweildauer auf (4,3 Jahre vs. 3,6 Jahre in der Vergleichsgruppe).

Von 242 Individuen weisen 7I (das entspricht $29 \%$ ) einen Hintergrund in einer sozialpartnerschaftlichen Organisation auf. Definiert wird dies als Amt oder Dienstverhältnis bei ÖGB, AK, WKO oder LWK zu einem Zeitpunkt vor Antritt des Regierungsamtes. Diese Informationen wurden auf Basis von Biografien der 
Regierungsmitglieder erhoben. ${ }^{9}$ Diese Angaben wurden eingehend studiert, jeder Verweis auf eine Funktion oder Anstellung in einer Sozialpartner-Organisation wurde festgehalten. Nicht gezählt wurden Funktionen in den Gewerkschafts- bzw. Kammerfraktionen (z. B. FunktionärInnen der ÖVP-Bünde oder der FSG). Da die Biografien von Regierungsmitgliedern in der Regel sehr umfangreich und detailgenau vorliegen, kann davon ausgegangen werden, dass darin sämtliche Funktionen und Dienstverhältnisse in Kammern und Gewerkschaften aufscheinen.

Prinzipiell wäre auch die Mitgliedschaft in Kammern oder Gewerkschaften ein möglicher Indikator. Jedoch sind etwa praktisch alle PolitikerInnen der SPÖ gleichzeitig Mitglied im ÖGB, wodurch eine empirische Differenzierung unmöglich wird. Die Mitgliedschaft in einer Kammer ist wiederum ein perfektes Korrelat des Berufes und somit als Indikator auch eher problematisch. Zu alldem kommt, dass Informationen über Mitgliedschaften in Kammern und Gewerkschaften in den Biografien der Regierungsmitglieder weit weniger gut abgebildet sind als politische Funktionen und Arbeitsverhältnisse.
Die Analyse beschränkt sich also auf die korporatistischen Kerninstitutionen - Kammern und Gewerkschaften. ${ }^{\text {Io }}$ Von den 72 identifizierten Personen hat ein Großteil Verbindungen zum ÖGB (32), gefolgt von Arbeiterkammer (20) und Wirtschaftskammer (I8), während nur sechs Personen aus dem Umfeld der Landwirtschaftskammern stammen (diese Zahlen ergeben in Summe mehr als 72, da einige Personen Verbindungen sowohl zum ÖGB als auch zur AK aufweisen).

Über die 27 Kabinette ${ }^{\text {II }}$ der Zweiten Republik betrachtet (siehe Abbildung I) gibt es interessante Variationen im Sozialpartner-Anteil an den Regierungsmitgliedern. Während der ersten zwanzig Jahre schwankt der Anteil zwischen einem Viertel und einem Drittel. Seinen Höhepunkt erreicht er im Kabinett Klaus I, dem letzten in der ersten Phase der großen Koalition. Während der Phase der Einparteienregierungen (1966 bis 1983) liegt der Anteil im Schnitt sogar noch geringfügig höher, besonders während der Kanzlerschaft von Bruno Kreisky.

Der Regierungseintritt der FPÖ im Jahr 1983 geht mit einem Rückgang des Sozialpartner-Anteils einher, jedoch folgt während der zweiten Phase der großen

Abbildung 1: Anteil der Regierungsmitglieder mit Sozialpartner-Hintergrund

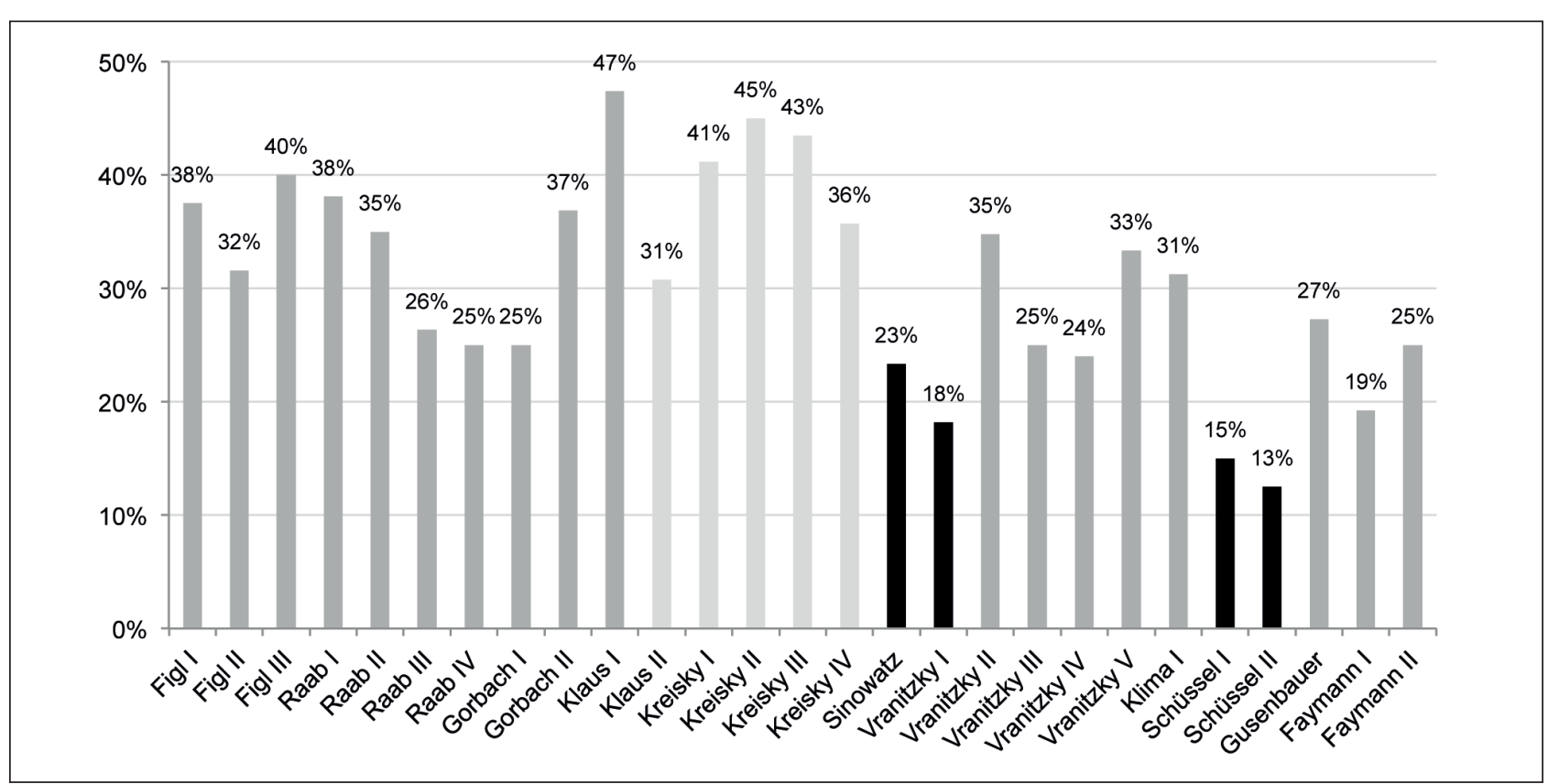

Anmerkung: Die Prozentzahlen geben den Anteil an allen MinisterInnen und Staatssekretärlnnen während der Regierungsperiode an. Hellgraue Balken markieren Einparteienregierungen, schwarze Balken markieren kleine Koalitionen.

9 Die zwei wichtigsten Quellen dabei waren das Internationale Biographische Archiv (www.munzinger.de) und die biografischen Angaben zu Regierungsmitgliedern auf den Internetseiten des österreichischen Parlaments (www.parlament.gv.at/WWER/). Das Internationale Biographische Archiv deckt den Großteil, die Parlamentsseiten die Gesamtheit aller relevanten Personen ab. In Einzelfällen wurden noch andere Quellen herangezogen, etwa das Weblexikon der Wiener Sozialdemokratie (www.dasrotewien.at) oder die Internetseiten der KPÖ-nahen Alfred Klahr Gesellschaft (www.klahrgesellschaft.at).
IO Daneben weisen vier Regierungsmitglieder Verbindungen zur Vereinigung der Österreichischen Industrie auf und sieben $\mathrm{zu}$ anderen Kammern (Rechtsanwaltskammer, Notariatskammer, Ärztekammer, Tierärztekammer, Landarbeiterkammer).

II Die Zählung der Kabinette orientiert sich an folgenden Kriterien: Neuwahlen, Kanzlerwechsel und Wechsel der Parteizusammensetzung (Ausnahme: der Wechsel von ÖVP-FPÖ zu ÖVP-BZÖ im Jahr 2005). 
Koalition (1987 bis 2000) ein Anstieg auf um die $30 \%$. Den niedrigsten Anteil an korporatistisch sozialisiertem Personal weisen die Kabinette Schüssel I und II auf. Mit Wiederauflage der großen Koalition unter den Kanzlern Gusenbauer und Faymann steigt der Sozialpartner-Anteil wieder an.

Wie aus Abbildung I ebenso hervorgeht, verzeichnen vor allem die kleinen Koalitionen unter Beteiligung der FPÖ einen Rückgang im Anteil der SozialpartnerFunktionärInnen. Dies ist natürlich zum Teil eine Folge dessen, dass die FPÖ über deutlich weniger Personal mit Verbindungen zu den Kammern und Gewerkschaften verfügt, zum Teil aber auch durch Verhaltensänderungen von SPÖ und ÖVP erklärbar. So sinkt auch innerhalb der ÖVP der Sozialpartner-Anteil unter Schüssel I und II auf ein Minimum, ${ }^{\text {I2 }}$ und auch die SPÖ ernennt zur Zeit der rot-blauen Koalition Mitte der I980er deutlich weniger Kammer- und Gewerkschaftsfunktionäre zu MinisterInnen als sonst.

\section{Analyse}

In Abbildung 2 wird ein erster Plausibilitätstest für die vier Hypothesen vorgenommen. Grundgesamtheit sind abermals die 242 Individuen. Wesentlich ist, dass hier nur die erste Ernennung jedes Individuums berücksichtigt wird, selbst wenn dieselbe Person im Nachfolgekabinett ebenfalls vertreten ist. Diese Zählweise ist hier notwendig, weil die oft hohe personelle Kontinuität über die Regierungen hinweg bei Mehrfachzählung zusätzliche Beobachtungen erzeugen würde, die aber keine unabhängigen Observationen darstellen. In der multivariaten Analyse (siehe unten) kann dieser Amtsinhabereffekt statistisch isoliert werden, deswegen wird dort jede Ernennung in jedem Kabinett einzeln berücksichtigt.

Abbildung 2 zeigt, dass es zwischen Regierungstypen große Unterschiede gibt. Allerdings liegen - entgegen den Erwartungen in $\mathrm{HI}$ - große Koalitionen und Einparteienregierungen beim Sozialpartner-Anteil in etwa gleichauf. Kleine Koalitionen haben erwartungsgemäß niedrigere Anteile.

Abbildung 2: Sozialpartner-Anteil nach Regierungstyp, Parteicharakteristika \& Ressort

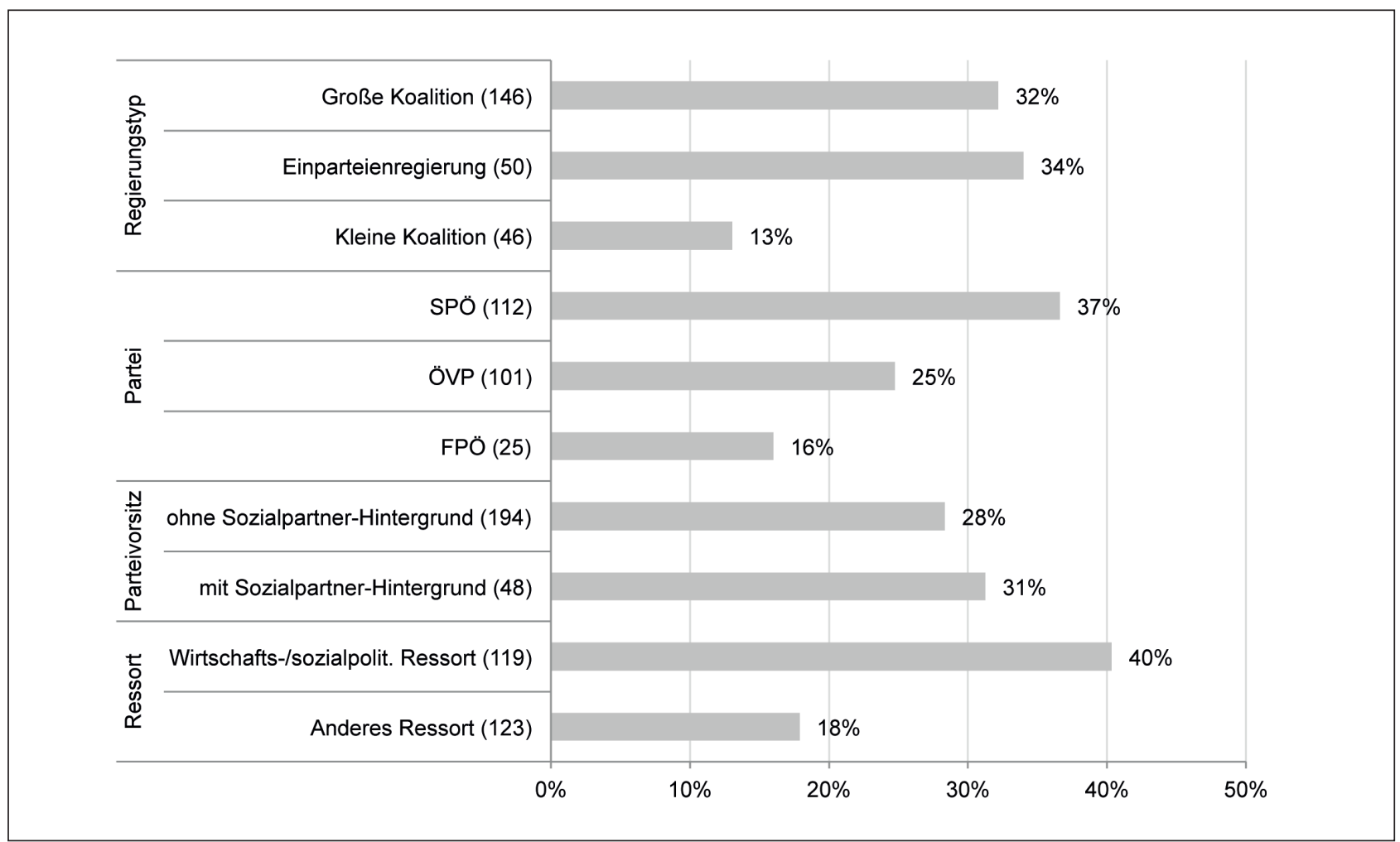

Anmerkung: Werte in Klammern geben die Anzahl Beobachtungen in der jeweiligen Kategorie an. Beispiel: Von 146 Personen, die unter großen Koalitionen erstmals ins Amt kamen, haben 32 \% Sozialpartner-Verbindungen. 
Betrachtet man die Ernennungen nach Parteien, ${ }^{13}$ so weist die SPÖ mit 37 \% den höchsten SozialpartnerAnteil auf, die ÖVP mit $25 \%$ den zweithöchsten und die FPÖ mit I6 \% den geringsten. Dieses Resultat ist im Einklang mit $\mathrm{H} 2$, zeigt aber auch, dass die Unterschiede zwischen den Parteien wohlmöglich nicht so deutlich sind wie angenommen. Bei Differenzierung nach Parteivorsitzenden mit und ohne Sozialpartner-Verbindung treten nur minimale Unterschiede zutage.

Große Differenzen sind hingegen bei der Unterscheidung zwischen wirtschafts- bzw. sozialpolitischen Ressorts und anderen Ministerien zu beobachten. In die Kategorie der wirtschafts- und sozialpolitischen Ressorts fallen alle Ministerien mit folgenden Zuständigkeiten: Arbeit, Bauten und Technik, Elektrifizierung und Energiewirtschaft, Finanzen, Gesundheit, Familie, Handel/ Gewerbe/Industrie, Konsumentenschutz, Land- und Forstwirtschaft, Soziales, Verkehr, Vermögenssicherung, verstaatlichte Betriebe, Volksernährung, Wiederaufbau und Wirtschaft. In diesen sozio-ökonomischen Ressorts haben rund 40 \% der Ernannten Sozialpartner-Verbindungen, wohingegen sich der Anteil in den verbleibenden Ressorts auf $18 \%$ beläuft, was den in $\mathrm{H}_{4}$ formulierten Erwartungen entspricht.

Um die vier Hypothesen systematisch überprüfen zu können, werden in der multivariaten Analyse mehrere logistische Regressionen geschätzt, dabei werden random effects auf Ebene der Kabinette spezifiziert. Die abhängige Variable ist ein dichotomer Indikator, der angibt, ob eine ernannte Person biografische Verbindungen zu einer Kammer oder Gewerkschaft ausweist. Analyseeinheit sind die Ernennungen pro Kabinett, was bedeutet, dass Personen mit Amtsdauern, die sich über mehrere Kabinette erstrecken, mehrmals vorkommen. Wenn Personen innerhalb einer Regierungsperiode von einem Regierungsamt in ein anderes wechseln, wird allerdings nur die erste Beobachtung inkludiert. Die Zahl der Beobachtungen steigt so auf $\mathrm{N}=572$.

Zusätzlich zu den unabhängigen Variablen für die Hypothesenüberprüfung werden zwei Kontrollvariablen inkludiert. Die Bedeutung des Ressorts wird mithilfe des Maßes für portfolio salience von Druckman und Warwick (2005) operationalisiert. Ressorts wie das Bundeskanzleramt, das Finanzministerium oder das $\mathrm{Au}-$ ßenministerium werden traditionell als wichtiger und prestigeträchtiger eingeschätzt als etwa Landwirtschaft, Sport oder einfache Staatssekretariate. Diese Varianz wird durch die portfolio salience-Variable erfasst. Des Wei-

I3 Nachdem das entscheidende Kriterium hier nicht die Parteimitgliedschaft der ernannten Personen ist, sondern die Partei, die für die Nominierung verantwortlich ist, wurden Parteilose soweit möglich den Parteien zugeschlagen, die das Vorschlagsrecht für die Position hatten. Bis auf drei Justizminister (Hans Kapfer, Egmont Foregger und Nikolaus Michalek) wurden so alle Individuen einer „ernennenden Partei“ zugeordnet, auch wenn sie selbst parteilos waren. teren wird - wie oben erwähnt - eine Variable zur Berücksichtigung von Wiederernennungen von MinisterInnen in aufeinanderfolgenden Kabinetten spezifiziert. Diese Variable ist dichotom und gibt an, ob die betreffende Position im vorangegangenen Kabinett schon mit einer Person mit Sozialpartner-Hintergrund besetzt war.

Tabelle 2 präsentiert fünf Regressionsmodelle. Gezeigt werden unstandardisierte Koeffizienten $(b)$, die durch Umformung $\left(e^{b}\right)$ in Odds Ratios umgerechnet werden können. Die Modellgüte, gemessen durch McFadden's korrigiertes Pseudo- $\mathrm{R}^{2}$, bewegt sich in fast allen Modellen um o,2, wobei der höchste Wert in Modell IV erreicht wird $(0,23)$. Modell IVa, das nur Ersternennungen heranzieht (d.h. sämtliche Wiederernennungen in aufeinanderfolgenden Kabinetten werden ausgeschlossen) erreicht eine deutlich niedrigere Modellgüte, was vor allem am hier logischerweise schwachen Effekt der Kontrollvariable für Amtsinhaber mit Sozialpartner-Hintergrund liegt.

In Modell I werden die Effekte der Regierungstypen getestet, hier zeigt sich ein negativer Effekt von kleinen Koalitionen, jedoch kein statistisch signifikanter Effekt von großen Koalitionen (Referenzkategorie sind Einparteienregierungen). In Modell II werden Parteieffekte geschätzt. Für die SPÖ ergibt sich ein positiver und statistisch signifikanter Effekt, jedoch nicht für die ÖVP. Hier stimmt die Richtung des Effekts zwar mit den Erwartungen überein, jedoch ist kein statistisch signifikanter Unterschied zur Referenzkategorie (= von anderen Parteien nominierte Minister, d.h. FPÖ, KPÖ, drei parteilose) festzustellen. In Modell III werden Ressortcharakteristika getestet, die hypothesenrelevante Variable zur Erfassung wirtschafts- und sozialpolitischer Ressort zeigt einen starken und statistisch signifikanten Effekt, der den Erwartungen von $\mathrm{H}_{4}$ entspricht. Die Bedeutung eines Ressorts (portfolio salience) hat hingegen keinen Effekt.

Die meisten dieser Effekte sind auch im kompletten Modell (IV) zu finden, allein der SPÖ-Effekt aus Modell II verschwindet. Das ist auch das bemerkenswerteste Ergebnis der multivariaten Analyse: Nach Kontrolle für Regierungstypen gibt es keine statistisch signifikanten Unterschiede zwischen den Parteien bei der Nominierung von Regierungsmitgliedern mit SozialpartnerHintergrund - ein Ergebnis, das angesichts der extrem starken Verankerung von SPÖ und ÖVP in den Gewerkschaften und Kammern zumindest auf den ersten Blick überraschend ist.

Auf den zweiten Blick zeigt sich, dass bestimmte Parteieffekte mit den Regierungstyp-Variablen schon erfasst werden - es handelt sich ja hier um Unterschiede zwischen Regierungen mit bestimmten Parteizusammensetzungen. Wie oben erwähnt legen SPÖ und ÖVP in kleinen Koalitionen andere Ernennungsmuster an den Tag. Unter den ÖVP-FPÖ/BZÖ-Regierungen 
Tabelle 2: Regressionsanalyse: Ernennung von Personen mit Sozialpartner-Hintergrund

\begin{tabular}{|c|c|c|c|c|c|}
\hline & I & II & III & IV & IVa \\
\hline \multirow[t]{2}{*}{ Kleine Koalition } & $-0.898^{*}$ & & & $-0.889^{*}$ & -0.991 \\
\hline & $(0.372)$ & & & $(0.431)$ & $(0.652)$ \\
\hline \multirow[t]{2}{*}{ Große Koalition } & 0.163 & & & 0.129 & 0.187 \\
\hline & $(0.265)$ & & & $(0.274)$ & $(0.376)$ \\
\hline \multirow[t]{2}{*}{ Partei: SPÖ } & & $1.198^{*}$ & & 0.724 & 0.579 \\
\hline & & $(0.506)$ & & $(0.575)$ & $(0.708)$ \\
\hline \multirow[t]{2}{*}{ Partei: ÖVP } & & $1.007 \#$ & & 0.435 & 0.0361 \\
\hline & & $(0.523)$ & & $(0.596)$ & $(0.729)$ \\
\hline \multirow[t]{2}{*}{ Parteivorsitzende/r mit Sozialpartner-Hintergrund } & & 0.259 & & 0.217 & 0.187 \\
\hline & & $(0.250)$ & & $(0.261)$ & $(0.373)$ \\
\hline \multirow[t]{2}{*}{ Wirtschafts-/sozialpolitisches Ressort } & & & $1.013^{* * *}$ & $1.132^{* * *}$ & $1.182^{\star \star \star}$ \\
\hline & & & $(0.220)$ & $(0.224)$ & $(0.310)$ \\
\hline \multirow[t]{2}{*}{ Bedeutung des Ressorts (portfolio salience) } & & & 0.366 & 0.320 & $0.630 \#$ \\
\hline & & & $(0.251)$ & $(0.255)$ & $(0.371)$ \\
\hline \multirow[t]{2}{*}{ Sozialpartner-Ressort im vorigen Kabinett } & $2.350^{* \star *}$ & $2.271^{\star * *}$ & $2.210^{\star * *}$ & $2.172^{* * *}$ & 0.294 \\
\hline & $(0.219)$ & $(0.239)$ & $(0.234)$ & $(0.226)$ & $(0.332)$ \\
\hline \multirow[t]{2}{*}{ Konstante } & $-1.762^{\star \star \star}$ & $-2.868^{\star * *}$ & $-2.611^{\star \star *}$ & $-3.182^{* * *}$ & $-2.582^{\star \star}$ \\
\hline & $(0.263)$ & $(0.497)$ & $(0.324)$ & $(0.673)$ & $(0.855)$ \\
\hline \multirow[t]{2}{*}{ Varianz (Kabinette) } & 0,000 & 0,0100 & 0,0851 & 0,000 & 0,000 \\
\hline & $(0,000)$ & $(0,0977)$ & $(0,114)$ & $(0,000)$ & $(0,000)$ \\
\hline Korr. Pseudo-R (McFadden) & 0,20 & 0,19 & 0,22 & 0,23 & 0,06 \\
\hline Log likelihood & -278 & -280 & -272 & -263 & -138 \\
\hline$N$ & 572 & 572 & 572 & 572 & 262 \\
\hline
\end{tabular}

Anmerkung: Präsentiert werden Koeffizienten logistischer Regressionsmodelle mit random effects auf Kabinettsebene, Standardfehler in Klammer; Modell IVa umfasst nur Ersternennungen, d.h. exklusive wiederholte Ernennungen in aufeinanderfolgenden Kabinetten; \# $p<0,1, * p<0,05, * * p<0,01$, $* * * p<0,001$.

war der Sozialpartner-Anteil etwa nicht nur wegen der Regierungsbeteiligung der FPÖ niedrig, sondern auch deshalb weil das ÖVP-Regierungsteam in diesen knapp sieben Jahren nur eine Person mit korporatistischem Hintergrund aufwies (Martin Bartenstein). Ähnliches gilt für die SPÖ-FPÖ-Koalitionen Mitte der I98oer-Jahre (wiewohl hier der Sozialpartner-Anteil aufgrund personeller Kontinuitäten aus den Kreisky-Kabinetten noch etwas höher lag).

Die deskriptive Statistik verstärkt dieses Bild: Während der Sozialpartner-Anteil in rot oder schwarz kontrollierten Ressorts unter großen Koalitionen bei $30 \%$ und in Einparteienregierungen gar bei 39 \% liegt, sinkt dieser Wert für kleine Koalitionen auf $18 \%$. Es ist also nicht allein der Regierungseintritt der Freiheitlichen, der für geringere Sozialpartner-Repräsentation in den Kabinetten kleiner Koalitionen sorgt, sondern auch das geänderte Verhalten der Großparteien. Eine mögliche Erklärung dafür wäre, dass Personen mit SozialpartnerHintergrund große Koalitionen bevorzugen, weil sie (zumindest eine Zeit lang) in diesem System von Verhandlungen zwischen SPÖ- und ÖVP-dominierten Verbänden sozialisiert wurden. Demgegenüber bevorzugen die Parteivorsitzenden in kleinen Koalitionen möglicherweise jenes Personal, das eher bereit ist, ein politisches Projekt abseits der Farben Rot und Schwarz mitzutragen.

Modell IV liefert also partielle Evidenz für $\mathrm{HI}(\mathrm{Re}-$ gierungstypen), jedoch keine für $\mathrm{H} 2$ (Parteien) und $\mathrm{H}_{3}$ 
(Parteivorsitzende). In Übereinstimmung mit $\mathrm{H}_{4}$ hat allerdings die Art des Ressorts entscheidenden Einfluss. Der Koeffizient für wirtschafts- und sozialpolitische Ministerien beträgt I.067, was einer Odds Ratio von rund 3.I entspricht. Somit steigt die Wahrscheinlichkeit einer Ernennung einer Person mit Sozialpartner-Verbindung in solchen Ministerien gegenüber anderen hier auf das mehr als dreifache.

Gesondert müssen wir Modell IVa betrachten. Hier ist die Analyse auf Ersternennungen beschränkt, d.h. Wiederernennungen derselben Person in aufeinanderfolgenden Kabinetten werden nicht berücksichtigt. Durch die geringere Fallzahl vergrößern sich für die meisten Koeffizienten die Standardfehler, wodurch etwa der Effekt für kleine Koalitionen nicht mehr statistisch signifikant ist $(p=0.126)$. Für die Variable Bedeutung des Ressorts erhöht sich aber auch der Koeffizient, wodurch der Effekt in Modell IVa erst signifikant positiv wird $(p=0.065)$.

Diesem Ergebnis wohnt eine intuitive Logik inne. Während bei Ersternennungen die Bedeutung des Ressorts eine gewisse Rolle spielt, dominiert danach der Amtsinhaber-Effekt gegenüber der portfolio salience. War eine Position in einem Kabinett mit einer sozialpartnerschaftlich sozialisierten Person besetzt, so ist die Wahrscheinlichkeit sehr hoch, dass dies im darauffolgenden Kabinett ebenso ist. Dieser Effekt kommt allein durch die politische Langlebigkeit vieler Amtsinhaber zustande, nicht durch „Erbpachten“ bestimmter Verbände auf bestimmte Ressorts. Dabei ist anzumerken, dass Sozial-
partner-FunktionärInnen in Regierungsämtern mit einem Median von 4,3 Jahren eine etwas längere Verweildauer aufweisen als Regierungsmitglieder (3,7 Jahre).

Die relevantesten substanziellen Ergebnisse (betreffend $\mathrm{HI}$ und $\mathrm{H}_{4}$ ) werden in Abbildung 3 mit vorhergesagten Wahrscheinlichkeiten dargestellt (geschätzt auf Basis von Modell IV). Die Wahrscheinlichkeit der Ernennung einer Person mit Sozialpartner-Hintergrund beträgt für große Koalitionen und Einparteienregierungen zwischen Io \% und I2 \%, sinkt aber für kleine Koalitionen auf rund $5 \%$. Noch größer fällt der Effekt für unterschiedliche Ressorts aus. Hier beträgt die vorhergesagte Wahrscheinlichkeit für wirtschafts- bzw. sozialpolitische Zuständigkeiten 29 \% gegenüber II \% für andere Ministerien. Bei all diesen Schätzungen sind die Unterschiede zwischen den Vergleichsgruppen relevanter als die absoluten Wahrscheinlichkeiten, da letztere stark davon abhängen, auf welchem Wert andere Variablen konstant gehalten werden.

Als Ergänzung zu den Hypothesentests stellt Tabelle 3 die empirischen Resultate einer nach Organisationen aufgegliederten Analyse dar. In Modell V wurde die abhängige Variable auf Fälle mit Zugehörigkeit zu ÖGB und AK begrenzt, während Modell VI die Ernennung von Personen mit biografischen Verbindungen zu WKO und LWK erfasst (eine feinere Aufgliederung ist aufgrund niedrigerer Fallzahlen wenig praktikabel).

Es zeigt sich, dass der Effekt von kleinen Koalitionen hauptsächlich für Personal mit Naheverhältnis zu ÖGB und AK auszumachen ist. Die Ernennung von

Abbildung 3: Vorhergesagte Wahrscheinlichkeiten für die Ernennung von Personen mit Sozialpartner-Hintergrund nach Regierungstyp und Ressort

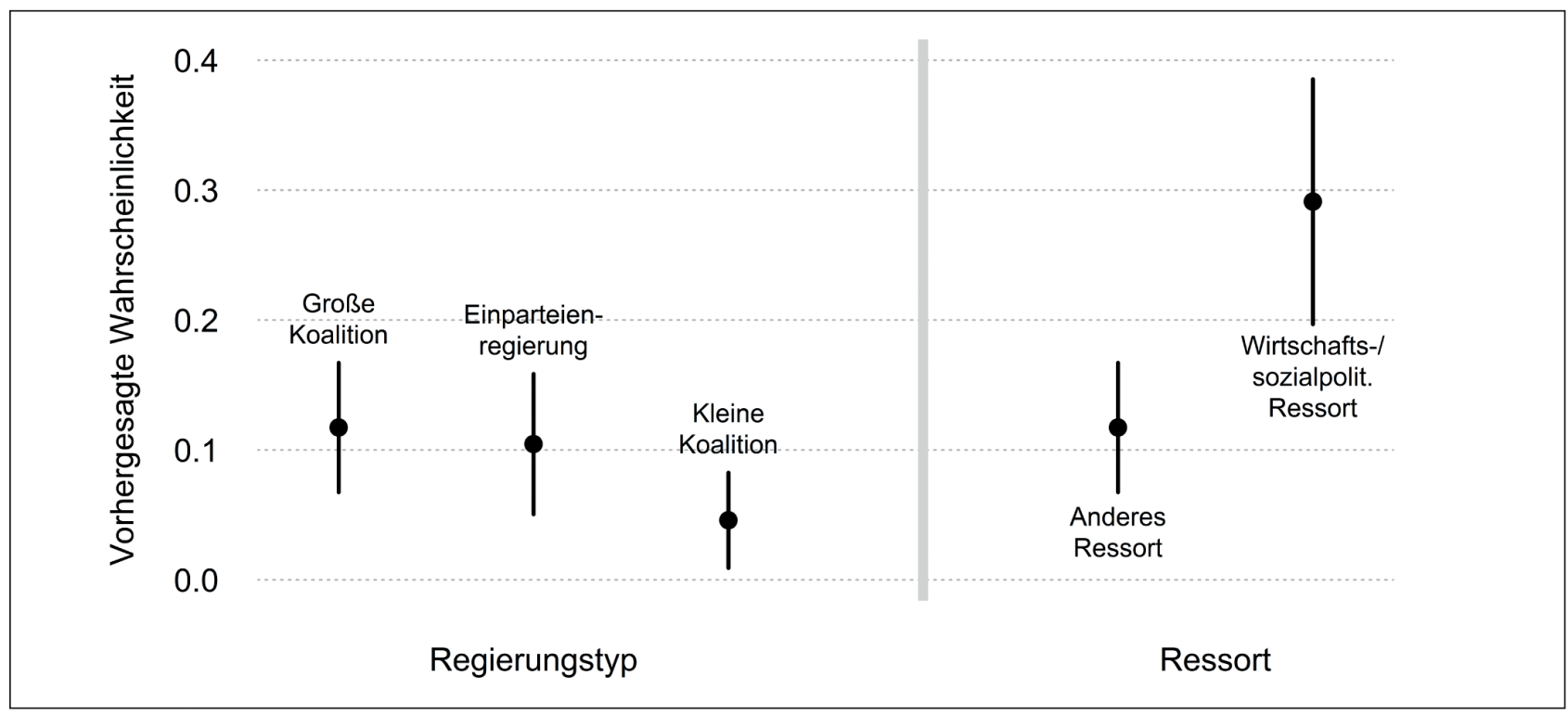

Anmerkung: Schätzungen basieren auf Modell IV (nur der fixed effects-Teil wird berücksichtigt). Alle dichotomen Variablen auf Modalwert konstant gehalten, alle kontinuierlichen Variablen auf Mittelwert. 
Tabelle 3: Regressionsanalyse: Ernennung von Personen mit Sozialpartner-Hintergrund, gegliedert nach Organisationszugehörigkeit.

\begin{tabular}{|c|c|c|}
\hline & v & VI \\
\hline & ÖGB/AK & WKO/LWK \\
\hline \multirow[t]{2}{*}{ Kleine Koalition } & $-0.897 \#$ & -0.121 \\
\hline & $(0.465)$ & $(0.837)$ \\
\hline \multirow[t]{2}{*}{ Große Koalition } & -0.112 & 0.758 \\
\hline & $(0.298)$ & $(0.532)$ \\
\hline \multirow[t]{2}{*}{ Partei: SPÖ } & $2.613^{*}$ & $-2.523^{* *}$ \\
\hline & $(1.079)$ & $(0.843)$ \\
\hline \multirow[t]{2}{*}{ Partei: ÖVP } & 0.448 & 0.328 \\
\hline & $(1.121)$ & $(0.776)$ \\
\hline \multirow[t]{2}{*}{ Parteivorsitzende/r mit Sozialpartner-Hintergrund } & 0.0420 & 0.533 \\
\hline & $(0.315)$ & $(0.388)$ \\
\hline \multirow[t]{2}{*}{ Wirtschafts-/sozialpolitisches Ressort } & $0.564^{*}$ & $1.636^{* * *}$ \\
\hline & $(0.256)$ & $(0.373)$ \\
\hline \multirow[t]{2}{*}{ Bedeutung des Ressorts (portfolio salience) } & 0.108 & 0.436 \\
\hline & $(0.299)$ & $(0.383)$ \\
\hline \multirow[t]{2}{*}{ Sozialpartner-Ressort im vorigen Kabinett } & $1.845^{* * *}$ & $1.824^{* * *}$ \\
\hline & $(0.255)$ & $(0.355)$ \\
\hline \multirow[t]{2}{*}{ Konstante } & $-4.367^{* * *}$ & $-4.463^{* * *}$ \\
\hline & $(1.150)$ & $(1.013)$ \\
\hline \multirow[t]{2}{*}{ Varianz (Kabinette) } & 0,000 & 0,000 \\
\hline & $(0,000)$ & $(0,000)$ \\
\hline Korr. Pseudo- $R^{2}$ (McFadden) & 0,25 & 0,29 \\
\hline Log likelihood & -205 & -129 \\
\hline N & 572 & 572 \\
\hline
\end{tabular}

Anmerkung: Präsentiert werden Koeffizienten logistischer Regressionsmodelle mit random effects auf Kabinettsebene, Standardfehler in Klammer; $\# p<0,1,{ }^{*} p<0,05,{ }^{* *} p<0,01,{ }^{* * *} p<0,001$.

MinisterInnen und StaatssekretärInnen mit WKO- oder LWK-Verbindung bleibt vom Regierungstyp praktisch unbeeinflusst.

Wenig überraschend tritt in den beiden Modellen in Tabelle 3 auch ein Partei-Effekt zutage. Die SPÖ ernennt mit markant höherer Wahrscheinlichkeit Personal aus ÖGB und AK, während sie deutlich weniger auf Personen mit Verbindung zu WKO und LWK zurückgreift. Dieses Muster entspricht den Naheverhältnissen der Kammern und Gewerkschaften zu den Parteien. Wie- wohl wenig überraschend, ist dieses Resultat doch eine wichtige Ergänzung zu den Ergebnissen mit Bezug auf H2. Während die Partei (nach Kontrolle für den Regierungstyp)wenig Einfluss auf die Wahrscheinlichkeit von Sozialpartner-Ernennungen hat, hat sie einen starken Einfluss darauf, woher die jeweiligen Personen kommen.

Die Modelle zeigen auch, dass der Effekt des Ressorttyps im WKO/LWK-Modell deutlich größer ist als im ÖGB/AK-Modell (die Koeffizienten unterscheiden sich mit $p<$ O.OoI). Das deutet darauf hin, dass Personen aus 
WKO und LWK spezialisierter eingesetzt werden. Von 24 Personen mit WKO/LWK-Hintergrund dienten nicht weniger als 15 (63\%) in den Ressorts Finanzen, Wirtschaft/Handel und Landwirtschaft. Von den 48 ÖGB/ AK-nahen Personen dienten immerhin I4 (29\%) im Sozialministerium, die anderen verteilen sich aber breit über eine Vielzahl an Ressorts.

Abgesehen von den Spezifika, die in Tabelle 3 gezeigt werden, bleiben für die Gesamtanalyse Regierungstyp und Ressort die wichtigsten substanziellen Erklärungsfaktoren für die Ernennung von Regierungsmitgliedern mit sozialpartnerschaftlicher Biografie. Zusätzliche Modellschätzungen zeigen, dass weder systematische Unterschiede zwischen MinisterInnen und StaatssekretärInnen noch eindeutige Zeittrends vorliegen. Weder lineare oder quadrierte Jahresvariablen noch individuelle Indikatoren für einzelne Dekaden zeigen in multivariaten Modellen statistisch signifikante Effekte. Daraus folgt, dass die Verflechtung zwischen Bundesregierung und Sozialpartnern in den 70 Jahren seit 1945 ausgesprochen stabil geblieben ist und keinem generellen Aufwärts- oder Abwärtstrend unterliegt. Wiewohl die Literatur vielerorts einen Bedeutungsrückgang für die Sozialpartnerschaft während der letzten drei Jahrzehnte diagnostiziert hat (Gerlich 1992; Karlhofer/Tálos 2000; Tálos 2006), so ist zumindest die Rekrutierung von Sozialpartner-FunktionärInnen in Regierungsämter davon unberührt geblieben.

\section{Schlussbetrachtung}

Wie der vorliegende Beitrag zeigt, ist die personelle Verflechtung zwischen sozialpartnerschaftlichen Organisationen und der Bundesregierung bis heute ein prägendes Charakteristikum für die Personalauswahl bei der Zusammensetzung der Bundesregierung. Sowohl in der SPÖ als auch in der ÖVP gibt es mit den ÖGB- bzw. Kammerfraktionen nach wie vor starke innerparteiliche Gruppen, die Repräsentanz auf Regierungsebene einfordern - und auch durchsetzen. Da diese Gruppen - nicht zuletzt aufgrund ihrer Verankerung in den Kammern und Gewerkschaften - über beträchtliche finanzielle und organisatorische Ressourcen verfügen, haben sie in der innerparteilichen Auseinandersetzung bis heute großes Gewicht. So gesehen ist die anhaltend hohe personelle Verflechtung zwischen Bundesregierung und Sozialpartnern zum Teil jedenfalls der organisatorischen Verschränkung zwischen Sozialpartnerschaft und Parteien über die Brückenköpfe der Gewerkschafts- bzw. Kammerfraktionen geschuldet.

Wie die Analyse zum ersten Mal zeigt, sinkt der Sozialpartner-Anteil beträchtlich in Regierungen mit FPÖ-Beteiligung - und zwar auch innerhalb der Regierungsriegen von SPÖ und ÖVP. Die genauen Gründe dafür sind mit den vorliegenden Daten empirisch nicht festzustellen, jedoch mag es sein, dass sozialpartnerschaftlich sozialisierte Personen durch ihre Erfahrungen im korporatistischen Milieu stärker großkoalitionär orientiert sind und daher im Rekrutierungsprozess weniger Berücksichtigung finden, wenn es darum geht, als Regierungsmitglieder das Projekt einer kleinen Koalition mitzutragen. ${ }^{14}$

Insgesamt zeigt die empirische Untersuchung aber, dass der hohe Verflechtungsgrad, den deskriptive Untersuchungen aus vergangenen Jahrzehnten festgestellt haben (Gerlich 1985; Marko 1992), bis heute ein Charakteristikum der Beziehungen zwischen Sozialpartnern und Regierung ist. Wie jedoch passt dieses Ergebnis zur Diskussion über den Niedergang der Sozialpartnerschaft (Pernicka/Hefler 2015; Tálos 2006; Tálos/Fink 2003; Tálos/Stromberger 2004)? Die vorliegende Untersuchung lässt zwei Möglichkeiten offen. So man die personelle Verflechtung zwischen Regierung und Sozialpartnern als allgemeinen Indikator für die Verfasstheit des Korporatismus in Österreich heranziehen will, wäre die Diagnose eines Niedergangs vorschnell - und in manchen Fällen wohl dem (temporären) Abkühlen der tripartistischen Beziehungen während der Kabinette Schüssel I und II geschuldet. Es ist aber genauso vorstellbar, dass die Stabilität der personellen Verflechtungen über eine Schwächung der sozialpartnerschaftlichen Beziehungen in anderen Dimensionen hinwegtäuscht. Zukünftige Untersuchungen könnten hier ansetzen und versuchen darzustellen, wie sehr personelle Verflechtung mit inhaltlichen Einflussmöglichkeiten in der Politikgestaltung einhergeht. Erst dann wird deutlich werden, in welchem Ausmaß personelle Verflechtung als Indikator Aussagekraft über die korporatistischen Beziehungen in Österreich insgesamt hat.

\section{Literatur}

Allern, Elin H/Nicholas Aylott/Flemming Juul Christiansen (2007). Social Democrats and Trade Unions in Scandinavia: The Decline and Persistence of Institutional Relationships, in: European Journal of Political Research, Vol. 46(5), 607-635.

Allern, Elin H/Tim Bale (2012). Political Parties and Interest groups: Disentangling Complex Relationships, in: Party Politics, Vol. I8(I), 7-25.

Andeweg, Rudy B (2000). Ministers as Double Agents? The Delegation Process between Cabinet and Ministers, in: European Journal of Political Research, Vol. 37(3), 377-395.

I4 Ein Beispiel für sozialpartnerschaftliche Skepsis gegenüber kleinen Koalitionen ist die ablehnende Haltung des Wirtschaftskammer-Präsidenten Christoph Leitl gegenüber der Neuauflage von Schwarz-Blau im Februar 2003. 
Anthonsen, Mette/Johannes Lindvall/Ulrich Schmidt-Hansen (201I). Social Democrats, Unions and Corporatism: Denmark and Sweden Compared, in: Party Politics, Vol. I7(I), II8-I34.

Aylott, Nicholas (2003). After the Divorce Social Democrats and Trade Unions in Sweden, in: Party Politics, Vol. 9(3), 369-390.

Bäck, Hanna/Marc Debus/Patrick Dumont (20II). Who gets What in Coalition Governments? Predictors of Portfolio Allocation in Parliamentary Democracies, in: European Journal of Political Research, Vol. 5O(4), 44I-478.

Blaschke, Sabine (1999). Gewerkschaftlicher Organisationsgrad in Österreich, in: Zeitschrift für Soziologie, Vol. 28(I), 60-7I.

Browne, Eric C/Karen Ann Feste (1975). Qualitative Dimensions of Coalition Payoffs: Evidence from European Party Governments, 1945-1970, in: American Behavioral Scientist, Vol. I8(4), 530-556.

Budge, Ian/Hans Keman (1990). Parties and Democracy: Coalition Formation and Government Functioning in Twenty States, Oxford.

Compston, Hugh (1994). Union Participation in Economic Policy-Making in Austria, Switzerland, The Netherlands, Belgium and Ireland, 1970-1992, in: West European Politics, Vol. I7(I), I23-I45.

Crepaz, Markus ML (1995). An Institutional Dinosaur: Austrian Corporatism in the Post-Industrial Age, in: West European Politics, Vol. I8(4), 64-88.

Dowding, Keith/Patrick Dumont (2009). Structural and Strategic Factors Affecting the Hiring and Firing of Ministers, in: dies. (Hg.): The Selection of Ministers in Europe: Hiring and Firing, London, $\mathrm{I}-2 \mathrm{O}$.

Dowding, Keith/Patrick Dumont (Hg.) (2015) The Selection of Ministers Around the World, London.

Druckman, James N/Paul V Warwick (2005). The Missing Piece: Measuring Portfolio Salience in Western European Parliamentary Emocracies, in: European Journal of Political Research, Vol. 44(I), I7-42.

Ecker, Alejandro/Thomas M Meyer/Wolfgang C Müller (2015). The Distribution of Individual Cabinet Positions in Coalition Governments: A Sequential Approach, in: European Journal of Political Research, Vol. 54(4), 802-818.

Ennser-Jedenastik, Laurenz (2013). Portfolio Allocation within Parties: The Role of Regional Party Branches, in: The Journal of Legislative Studies, Vol. I9(3), 309327.

Falkner, Gerda/Simone Leiber (2004). Europeanization of Social Partnership in Smaller European Democracies?, in: European Journal of Industrial Relations, Vol. Io(3), 245-266.

Fink, Marcel (2006). Unternehmerverbände, in Herbert Dachs/Peter Gerlich/Herbert Gottweis/Helmut Kramer/ Volkmar Lauber/Wolfang C Müller/Emmerich Tálos
(Hg.): Politik in Österreich. Das Handbuch, Wien, 443-46I.

Gerlich, Peter (1985). Sozialpartnerschaft und Regierungssystem, in Peter Gerlich/Edgar Grande/Wolfgang C Müller (Hg.): Sozialpartnerschaft in der Krise: Leistungen und Grenzen des Neokorporatismus in Österreich, Wien, I09-I33.

Gerlich, Peter (1992). A Farewell to Corporatism, in: West European Politics Vol. I5(I), I32-I46.

Gerlich, Peter/Edgar Grande/Wolfgang C Müller (Hg.) (1985). Sozialpartnerschaft in der Krise: Leistungen und Grenzen des Neokorporatismus in Österreich, Wien.

Heinisch, Reinhard (2000). Coping with Economic Integration: Corporatist Strategies in Germany and Austria in the 1990s, in: West European Politics, Vol. 23(3), 67-96.

Indridason, Indridi H/Christopher Kam (2008). Cabinet Reshuffles and Ministerial Drift, in: British Journal of Political Science, Vol. 38(4), 62 I-656.

Kam, Christopher/William T Bianco/Itai Sened/Regina Smyth (2010). Ministerial Selection and Intraparty Organization in the Contemporary British Parliament, in: American Political Science Review, Vol. IO4(2), 289-306.

Karlhofer, Ferdinand (2006). Arbeitnehmerorganisationen, in Herbert Dachs/Peter Gerlich/Herbert Gottweis/Helmut Kramer/Volkmar Lauber/Wolfang C. Müller/Emmerich Tálos (Hg.): Politik in Österreich. Das Handbuch, Wien, 462-479.

Karlhofer, Ferdinand/Emmerich Tálos (1996). Sozialpartnerschaft und EU, Wien.

Karlhofer, Ferdinand/Emmerich Tálos (2000). Sozialpartnerschaft unter Druck. Trends und Szenarien, in: Anton Pelinka/Fritz Plasser/Wolfgang Meixner (Hg.): Die Zukunft der österreichischen Demokratie. Trends, Prognosen und Szenarien, Wien, 38I-4O2

Kenworthy, Lane (2003). Quantitative Indicators of Corporatism, in: International Journal of Sociology, Vol. 33(3), IO-44.

Kittel, Bernhard (1996). Sozialpartnerschaft und Disorganisierung: Veränderung und Kontinuität der Entscheidungsstrukturen in der österreichischen $\mathrm{Ar}$ beitszeitpolitik, in: Swiss Political Science Review, Vol. 2(4), I-26.

Kittel, Bernhard (2000). Deaustrification? The Policyarea-Specific Evolution of Austrian Social Partnership, in: West European Politics, Vol. 23(I), IO8-I29.

Krings, Torben (2013). Von der „Ausländerbeschäftigung“ zur Rot-Weiß-Rot-Karte: Sozialpartnerschaft und Migrationspolitik in Österreich, in: Österreichische Zeitschrift für Politikwissenschaft, Vol. 42(3), 263278.

Lehmbruch, Gerhard (1984). Interorganisatorische Verflechtungen im Neokorporatismus, in: Jürgen W 
Falter/Christian Fenner/Michael T Greven (Hg.): Politische Willensbildung und Interessenvermittlung, Opladen, 467-482.

Lehmbruch, Gerhard (1985). Sozialpartnerschaft in der vergleichenden Politikforschung, in: Peter Gerlich/ Edgar Grande/Wolfgang C Müller (Hg.): Sozialpartnerschaft in der Krise: Leistungen und Grenzen des Neokorporatismus in Österreich, Wien, 85-IO7.

Lijphart, Arend (1999). Patterns of Democracy: Government Forms and Performance in Thirty-Six Countries, New Haven.

Marko, Joseph (1992). Verbände und Sozialpartnerschaft, in: Wolfgang Mantl (Hg.): Politik in Österreich. Die Zweite Republik: Bestand und Wandel, Wien, 429478.

Matis, Herbert/Dieter Stiefel (1982). Der österreichische Abgeordnete. Der österreichische Nationalrat 19191979: Versuch einer historischen Kollektivbiographie, Wien.

Müller, Wolfgang C (1985). Die Rolle der Parteien bei der Entstehung und Entwicklung der Sozialpartnerschaft: Eine handlungslogische und empirische Analyse, in: Peter Gerlich/Edgar Grande/Wolfgang C Müller (Hg.): Sozialpartnerschaft in der Krise: Leistungen und Grenzen des Neokorporatismus in Österreich, Wien, 135-224.

Müller, Wolfgang C (1994). The Development of Austrian Party Organizations in the Post-War Period, in: Richard S Katz/Peter Mair (Hg.): How Parties Organize: Change and Adaptation in Party Organizations in Western Democracies, London, 5I-79.

Müller, Wolfgang C/Wilfried Philipp/Barbara Steininger (1994). Political Elites in Austria: Stability, „Interlocking Directorates" and the Challenge of Popular Disenchantment, Madrid.

Obinger, Herbert (2009). Sozialpolitische Bilanz der Großen Koalition in Österreich, in: Herbert Obinger/ Elmar Rieger (Hg.): Wohlfahrtsstaatlichkeit in entwickelten Demokratien: Herausforderungen, Reformen und Perspektiven, Frankfurt/Main,

Otjes, Simon/Anne Rasmussen (2015). The Collaboration between Interest Groups and Political Parties in Multi-Party Democracies: Party System Dynamics and the Effect of Power and Ideology, in: Party Politics, Vol. 23(2), 96-IO9, DOI: IO.II77/I3540688I4568046.

Pelinka, Anton (198I). Modellfall Österreich? Möglichkeiten und Grenzen der Sozialpartnerschaft, Wien.

Pernicka, Susanne/Günter Hefler (2015). Austrian Corporatism - Erosion or Resilience?, in: Österreichische Zeitschrift für Politikwissenschaft Vol. 44(3), 40-56.

Prischnig, Manfred (I99I). Bestandsaufnahme der Sozialpartnerschaft, in: Wirtschaft und Gesellschaft, Vol. I7(I), 9-36.
Rose, Richard (2000). The End of Consensus in Austria and Switzerland, in: Journal of Democracy, Vol. II(2), 26-40.

Schäfer, Armin/Wolfgang Streeck (2008). Korporatismus in der Europäischen Union, in: Martin Höpfner/Armin Schäfer (Hg.): Die politische Ökonomie der europäischen Integration, Frankfurt, 203-240.

Schwediauer, Veronika (2010). Same Same But Different: The Political Recruitment of Women and Men to the Austrian Government, Wien.

Siaroff, Alan (1999). Corporatism in 24 Industrial Democracies: Meaning and Measurement, in: European Journal of Political Research, Vol. 36(2), 175-205.

Sickinger, Hubert (2009). Politikfinanzierung in Österreich, Wien.

Tálos, Emmerich (1985). Sozialpartnerschaft: Zur Entwicklung und Entwicklungsdynamik kooperativkonzertierter Politik in Österreich, in: Peter Gerlich/ Edgar Grande/Wolfgang C Müller (Hg.): Sozialpartnerschaft in der Krise. Leistungen und Grenzen des Neokorporatismus in Österreich, Wien, 42-83.

Tálos, Emmerich (2006). Sozialpartnerschaft. Austrokorporatismus am Ende?, in: Herbert Dachs/Peter Gerlich/Herbert Gottweis/Helmut Kramer/Volkmar Lauber/Wolfang C Müller/Emmerich Tálos (Hg.): Politik in Österreich. Das Handbuch, Wien, 425-442.

Tálos, Emmerich (2008). Sozialpartnerschaft. Ein zentraler Gestaltungsfaktor der zweiten Republik, Innsbruck.

Tálos, Emmerich/Marcel Fink (2003). Sozialpartnerschaft in Österreich: Das korporatistische Modell am Ende?, in: Sven Jochem/Nico A Siegel (Hg.): Konzertierung, Verhandlungsdemokratie und Reformpolitik im Wohlfahrtsstaat: Das Modell Deutschland im Vergleich, Wiesbaden, I94-23I.

Tálos, Emmerich/Bernhard Kittel (200I). Gesetzgebung in Österreich. Netzwerke, Akteure und Interaktionen in politischen Entscheidungsprozessen, Wien.

Tálos, Emmerich/Christian Stromberger (2004). Verhandlungsdemokratische Willensbildung und korporatistische Entscheidungsfindung am Ende? Einschneidende Veränderungen am Beispiel der Gestaltung des österreichischen Arbeitsrechtes, in: Österreichische Zeitschrift für Politikwissenschaft, Vol. 33(2), I57-I74.

Traxler, Franz (1987). Klassenstruktur, Korporatismus und Krise: Zur Machtverteilung in Österreichs „Sozialpartnerschaft" im Umbruch des Weltmarkts, in: Politische Vierteljahresschrift, Vol. 28(I), 59-79. 


\section{Autor}

Laurenz Ennser-Jedenastik ist Universitätsassistent am Institut für Staatswissenschaft der Universität Wien. Er lehrt und forscht dort zu Parteien, Wahlen, Regierungen, politischen Eliten, Bürokratie und Sozialpolitik. Seine Beiträge wurden unter anderem in Comparative Political Studies, European Journal of Political Research, Journal of Public Administration Research and Theory, Party Politics, West European Politics und International Journal of Press/ Politics veröffentlicht. 